\title{
Methods for introducing carbon into the composition of structural powder materials
}

\author{
Maxim Egorov ${ }^{1 *}$, Rimma Egorova ${ }^{1}$, and Zanna Ereveeva ${ }^{2}$ \\ ${ }^{1}$ Don State Technical University, 34400, Gagarin Square 1, Rostov-on-Don, Russia \\ ${ }^{2}$ MISiS National University of Science and Technology, 119991, Leninsky prospect, 4 Moscow, \\ Russia
}

\begin{abstract}
Durability and reliability of machines and mechanisms depends on the structural strength of the products from which they are made. The main requirement for any product is trouble-free operation under the conditions of the established operational period. This determines the requirements for structural materials, the main of which are technological, operational and economic. The production of sintered powder products ensures the lowest energy consumption and the lowest waste. The introduction of carbon into powder materials significantly increases the strength characteristics of the product.
\end{abstract}

The strength characteristics of sintered iron-carbon materials are a function of the manufacturing conditions and the composition of the feedstock. To obtain iron-carbon materials, different raw materials can be used: a mixture of iron and graphite powders, a mixture of iron and cast iron powders, powder of pulverized carbon steel. Table 1 shows data characterizing the strength of carbon steel obtained by powder metallurgy methods from various types of raw materials $[1,2,3]$.

Table 1. Strength characteristics of carbon steel obtained from various raw materials. Porosity $15 \%$, carbon content $0.8 \%$.

\begin{tabular}{|c|c|c|c|c|c|c|}
\hline \multirow{3}{*}{$\begin{array}{l}\text { The composition of the } \\
\text { charge }\end{array}$} & \multirow{3}{*}{$\begin{array}{l}\text { Temper } \\
\text { ature } \\
\text { sintering } \\
,{ }^{\circ} \mathrm{C}\end{array}$} & \multicolumn{4}{|c|}{ Mechanical properties, $\mathrm{kg} / \mathrm{mm}^{2}$} & \multirow{3}{*}{ Structure } \\
\hline & & \multicolumn{2}{|c|}{ After sintering } & \multicolumn{2}{|c|}{$\begin{array}{l}\text { After quenching } \\
\text { and leave }\end{array}$} & \\
\hline & & $\sigma_{\mathrm{B}}$ & $\sigma_{\text {изг }}$ & $\sigma_{\mathrm{B}}$ & $\sigma_{\text {изг }}$ & \\
\hline Iron $+1 \%$ graphite & 1100 & 18 & 43 & 45 & 60 & $\mathrm{P}+\mathrm{C}+\mathrm{F}$ \\
\hline $\begin{array}{c}\text { Iron }+3 \% \\
\text { white cast iron powder }\end{array}$ & 1150 & 33 & 68 & 60 & 90 & $\mathrm{P}+\mathrm{C}$ \\
\hline Eutectoid Steel Powder & 1050 & 37 & 75 & 68 & 105 & $\mathrm{P}$ \\
\hline
\end{tabular}

Designations: $\mathrm{P}$ - perlite, $\mathrm{C}$ - cementite, $\mathrm{F}$ - ferrite.

\footnotetext{
*Corresponding author: aquavdonsk@mail.ru
} 
The method of manufacturing carbonaceous materials by sintering compacts from a mixture of iron and graphite has two fundamental drawbacks: the difficulty in obtaining a homogeneous charge and a high ratio of the volume of iron to the volume of graphite (32: 1), which significantly increases the diffusion path of carbon. These disadvantages lead to the fact that instead of the desired pearlite structure, a structure containing a large amount of cementite and ferrite is often obtained, and very unstable properties.

The indicated disadvantages of iron-graphite charges can be eliminated by using a mixture of iron powder with white cast iron powder, which is a waste product from the production of cast iron shot and is 15 times cheaper than iron powder and graphite. In this case, the ratio of the volume of iron to the volume of the carbon-containing component is only 2.3: 1 . To obtain these materials with an equilibrium structure, a sintering temperature of $1100-1150{ }^{\circ} \mathrm{C}$ is sufficient [2,3,4].

The advantage of this type of introduction of carbon into the composition of irongraphite materials is explained by the fact that at $1100^{\circ} \mathrm{C}$ the diffusion coefficient of carbon from cast iron particles is 1.5 times higher than from graphite, with approximately the same activation energy (Table 2).

Table 2. The value of the diffusion coefficient of carbon in austenite.

\begin{tabular}{|l|l|l|}
\hline \multirow{2}{*}{ Temperature, ${ }^{\circ} \mathrm{C}$} & \multicolumn{2}{|l|}{ Carbon diffusion coefficient, $\mathrm{cm}^{2} / \mathrm{sec}$} \\
\cline { 2 - 3 } & Cast iron-iron & Graphite-iron \\
\hline \multirow{2}{*}{1173} & $0,8 \cdot 10^{-7}$ & $4,9 \cdot 10^{-8}$ \\
1273 & $2,8 \cdot 10^{-7}$ & $1,6 \cdot 10^{-7}$ \\
1373 & $6,4 \cdot 10^{-7}$ & $4,4 \cdot 10^{-7}$ \\
1473 & $1,4 \cdot 10^{-6}$ & $1,0 \cdot 10^{-6}$ \\
& & 33500 \\
Activation energy, & 3450 & \\
cal / mol & & \multicolumn{2}{|l}{} \\
\hline
\end{tabular}

Acceleration of diffusion processes makes it possible to obtain, in the case of using cast iron powder as a carburizing component, a more uniform structure and higher strength of the material.

The most equilibrium structure and the highest level of strength and properties of sintered steel can be achieved using powder of eutectoid steel obtained by spraying the melt. In this case, each particle is an alloy and no heterodiffusion processes occur during sintering.

Another way of introducing carbon into the composition of iron-based structural materials is carburizing - chemical heat treatment, when carbon comes from a solid or gaseous carburizer [5,6]. The most commonly used solid carburizer is charcoal, and the backfill, into which the products are placed, is a mixture of charcoal with $20-25 \% \mathrm{~B} \alpha C O_{3}$ and $2-3 \% \mathrm{C} \alpha \mathrm{CO}_{3}$.

Cementation is carried out at a temperature of $950-1000^{\circ} \mathrm{C}$, in some cases it is combined with sintering. A feature of this method of introducing carbon into the composition of iron-based materials is the uneven composition of the carburized layer and its limited thickness.

Sintering of iron-graphite pressed billets. The introduction of carbon in the form of graphite into powder steels increases their strength, but reduces shrinkage and lowers toughness.

When sintering iron-graphite products, depending on the content of graphite in the powder mixture, the sintering temperature is in the range of $1050-1200^{\circ} \mathrm{C}$. According to 
the iron-carbon phase diagram (Fig. 1), up to $2.14 \%$ carbon can be dissolved in the structure of $\gamma$-iron in this temperature range.

Subsequent cooling of sintered products from the austenite region (depending on the content of dissolved carbon in austenite) should be accompanied by the release of ferrite along the GOS line, and cementite along the SE line and the eutectoid transformation of the remaining austenite into pearlite at the Ar1 point temperature.

As a result of these transformations, the final structure of sintered articles made of irongraphite should consist of ferrite and cementite, the quantitative ratio of which is determined by the carbon content in austenite.

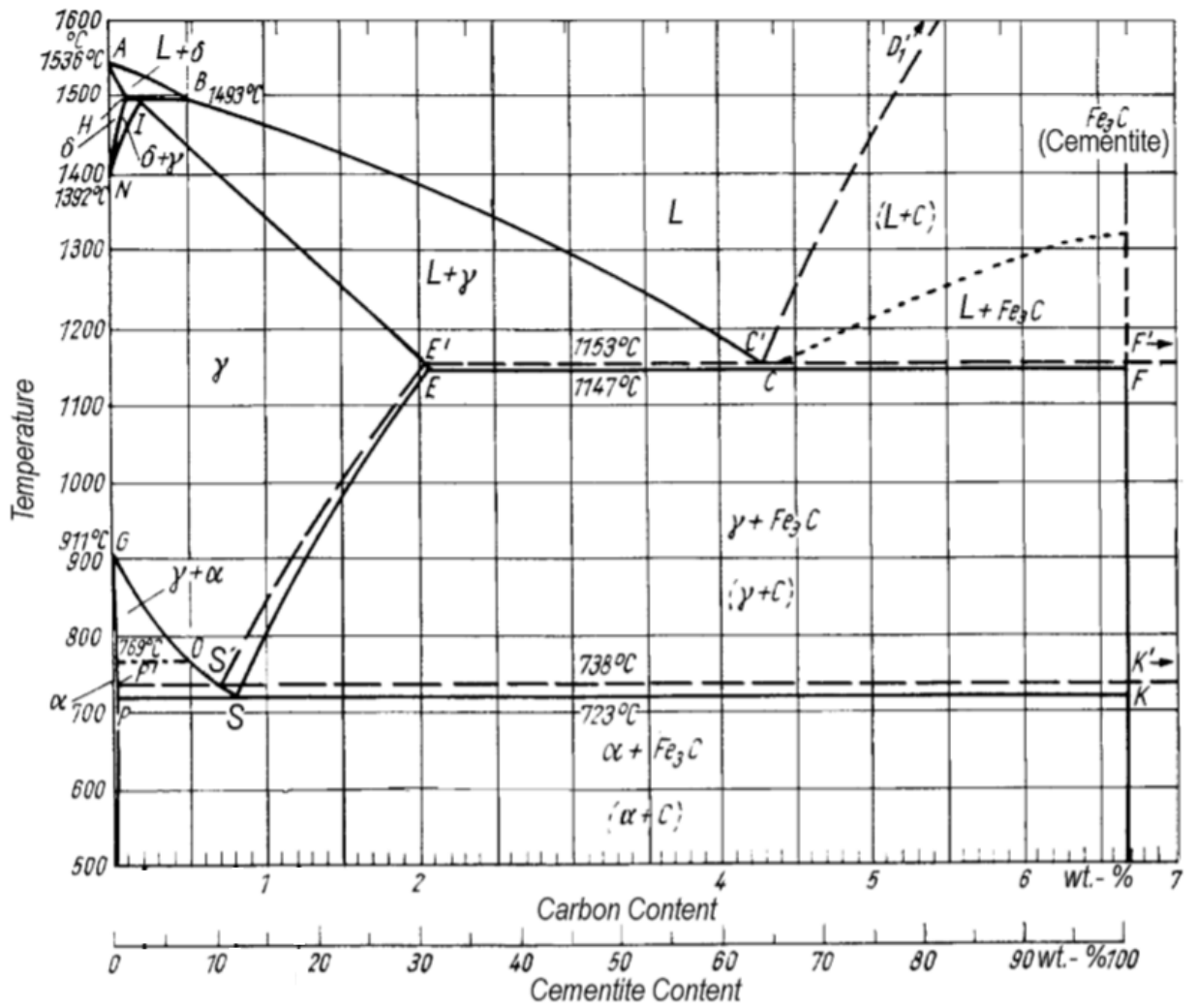

Fig. 1. State diagram of the iron-carbon system.

The resulting new phase - cementite - has a larger volume than ferrite and therefore leads to a decrease in the actual shrinkage of steel powder products during sintering, depending on the carbon content in them and the initial porosity of the pressed blanks, the following formula can be used:

Where:

$$
\frac{\Delta V_{c}}{V_{0}}=\frac{\Delta V_{0}}{V_{0}}-1,3 \frac{\% C}{\% F e}\left(\frac{100-\Pi_{0}}{100}\right)
$$

$\Delta \mathrm{Vc} / \mathrm{V} 0$ is the relative shrinkage of iron-graphite products;

$\Delta \mathrm{V} / \Delta \mathrm{V} 0$ - relative shrinkage of products made of graphite-free powder;

$\Pi_{0}$ - porosity of the pressed billet, volume fractions $\%, \% \mathrm{Fe}$ and\% $\mathrm{C}$ - the content of iron and carbon in the volume of the sintered product. 
It follows from the formula that an increase in the carbon content in the product reduces shrinkage, and an increase in porosity activates it. Figure 2 shows the calculated and experimental data of the dependence of the shrinkage on the carbon content, and the porosity of sintered specimens from reduced iron powder of grade PZh4M2 with different contents of graphite in the mixture (specimens were sintered at $1100^{\circ} \mathrm{C}$ for $3 \mathrm{~h}$ in an atmosphere of dried and purified nitrogen, in containers filled with a mixture of graphite grit and calcined alumina).

Comparison of the calculated by the formula and experimental data indicates good agreement. A somewhat overestimated arrangement of the experimental points characterizes the activation of the sintering process in the presence of graphite in the mixture.

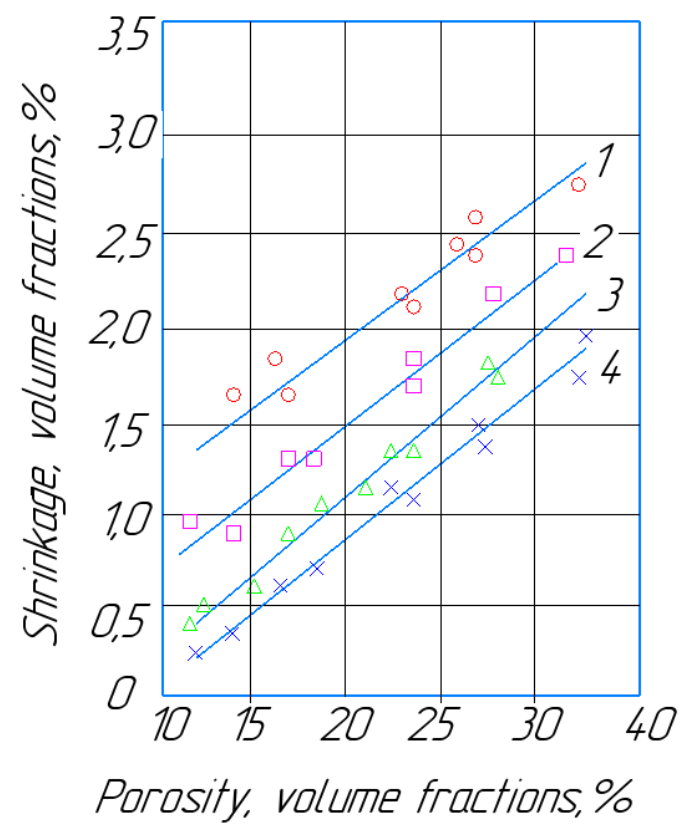

Fig. 2. Shrinkage depending on the porosity of the samples, determined by calculation, for samples with a carbon content, respectively: $1-0.1-0.2 ; 2-0.4-0.43 ; 3-0.8-0.82 ; 4-1.0-1.12 \%$ (solid lines); the obtained experimental data are indicated by icons

Porosity and sintering time have a great influence on shrinkage and structure formation during sintering of iron-graphite products. Thus, it follows from Fig. 3 that, with a short sintering time of specimens with 5\% pores, specimens that do not contain graphite have the greatest shrinkage (Fig. 3, curve 1). At porosity above 10 - 12\%, on the contrary, specimens with graphite have the greatest shrinkage (Fig. 3, a, curves 2 - 4).

The study of shrinkage depending on the sintering time of samples with a porosity of 15$17 \%$ showed that an increase in the sintering time changes the nature of the shrinkage dependence: the longer the sintering time, the predominant is the shrinkage of products without graphite (Figure 3, b). Thus, during sintering for $190 \mathrm{~min}$, the shrinkage of specimens without graphite is $1.3-1.4$ times greater than that of specimens with $1.4 \%$ graphite [7]. 


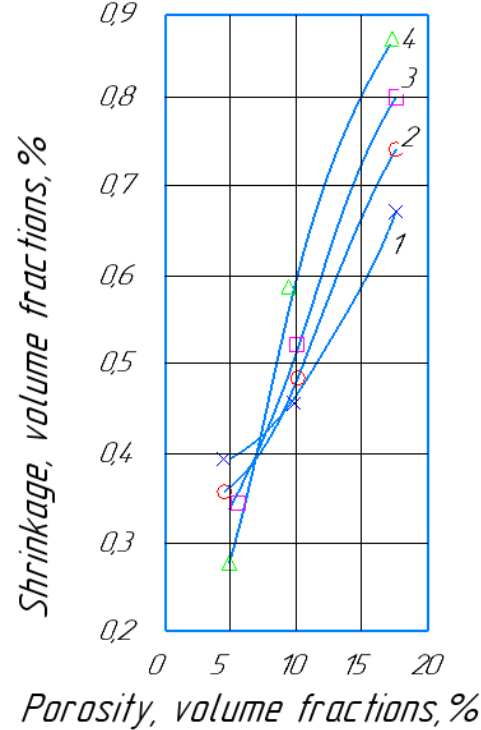

a)

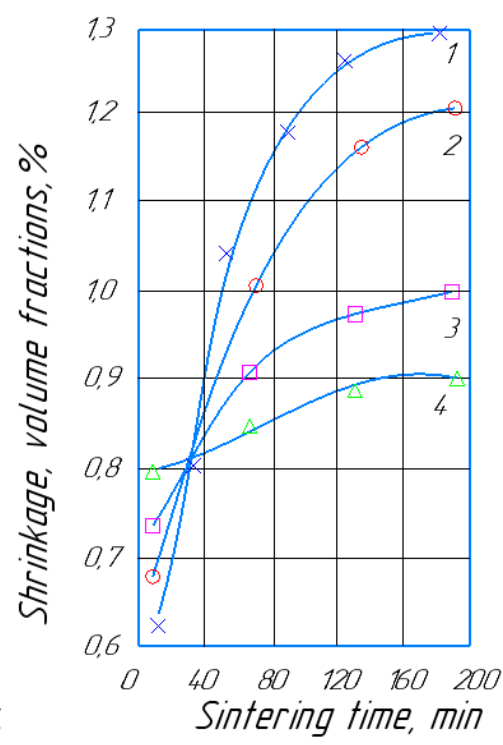

b)

Fig. 3. Shrinkage of specimens sintered at $1150^{\circ} \mathrm{C}$ in an atmosphere of dissociated ammonia depending on: a - on porosity; b - sintering time (porosity 14 - 16\%); samples were made: 1 - from PZh4M2 powder; 2 - 4 - from PZh4M2 powder with additions of $0.5,1.0$ and 1.4\% graphite, respectively

During heating and isothermal holding during sintering, there is a continuous interaction of the surface layers of iron particles with graphite both directly through the contact areas of graphite particles with iron particles and through the gas phase. Due to the fact that graphite (carbon) is an active reducing agent of iron oxides, during heating above 500-600 ${ }^{\circ}$ $\mathrm{C}$, reduction reactions begin to occur at the points of contact of graphite particles with iron, which contributes to the formation of a metal contact, an increase in self-diffusion of iron atoms and an increase in shrinkage [4,5,6].

At the same time, due to the reaction between the particles of graphite and iron, graphite with the atmosphere of the furnace, conditions for the formation of a carbon-containing gaseous medium are created. Since the kinetics of chemical reactions at the metal-gas interface is much higher than the diffusion coefficients, then the reduction processes are intensified not only in the contact areas of iron particles with graphite, but also over the entire surface of particles and pore surfaces communicating with the furnace atmosphere.

At the same time, diffusion of carbon into iron particles begins to develop through the metal contacts formed on the surface of the particles. Due to the fact that the dissolution of carbon in $\alpha$-iron is insignificant, and diffusion in ferrite proceeds slowly, before the $\alpha \rightarrow \gamma$ transformation, the most probable is the formation of cementite at the contact points as a phase with a more significant diffusion coefficient of carbon at these temperatures. The formation of cementite in interparticle metal contacts should inhibit shrinkage.

An abnormality is observed in the structure of powder steels. So, in the structure of SP80 steel, along with pearlite, colonies of ferrite and cementite can be found. This is due to the fact that graphite, differing in physical properties from other components of the mixture, is unevenly distributed in it, which leads to abnormal formation of structural components during sintering. The abnormality of the structural state of powder steels obtained on the basis of mechanical mixtures of components increases with the complication of the composition of the mixture by components. The presence of interparticle boundaries, 
making it difficult to redistribute carbon, also contributes to the appearance of abnormality in the structure $[1,3,5,8]$.

In the places of contact of $\mathrm{Fe}$ and $\mathrm{C}$ particles, as a result of direct chemical interaction, structurally free "primary" cementite is formed. As a result, the structure of sintered steel containing $2 \% \mathrm{C}$ consists of pearlite, a mesh of ledeburite eutectic, small inclusions of ferrite, undissolved particles of $\mathrm{C}$ in $\mathrm{Fe}-\mathrm{C}$ compositions, the whole spectrum of structures is often observed - pearlite, ferrite, cementite, ledeburite, graphite, which is not in cast materials.

The degree of abnormality in the structure of Fe - C materials depends on the amount, grade, size, and purity of the initial powders, on the sintering temperature, and on the cooling rate.

The observed reduced shrinkage during sintering of products with low porosity (4-8\%) is associated with the release of products of reduction of oxides by graphite, which (when leaving the volume of the product) loosen it; short sintering time (10 min, see Fig. 3, a, curves 2 - 4) does not provide shrinkage due to other processes. A decrease in shrinkage with an increase in sintering time is explained by the dissolution of graphite in $\gamma$-iron and the formation of cementite upon cooling, the presence of which increases the volume of the product and reduces shrinkage (see Fig. 3, b).

Thus, it was found that the introduction of graphite into the powder mixture activates sintering. The retarding effect of graphite can manifest itself only when its content in the powder mixture is greater than the possible limit of solubility of carbon in $\gamma$-iron at a given sintering temperature.

\section{References}

[1] S. Ivanov Study of the influence of the nature of nanosized particles and the mixing method on the mechanical properties of powder alloy steel PK70H3. Nanotechnology: Science and Manufacturing. (2015)

[2] Yu. Milman Mechanical properties of sintered materials. The effect of porosity on the plasticity of powder alloys. Powder metallurgy. (1991)

[3] V. Lopatin, J. Eremeeva Powder metallurgy in the automotive industry and other areas of industry: a tutorial. University of Mechanical Engineering (2014)

[4] G. Lieberson, V. Lopatin. Powder metallurgy processes MISIS 2002.

[5] R. Egorova Technology of manufacturing parts by powder metallurgy method and advanced materials used in additive technologies of DSTU (2020)

[6] R. Egorova Modern technological processes of powder metallurgy DSTU (2021)

[7] L. Dyachkova Influence of nanodispersed additives on the structure and properties of powder carbon and high-chromium steel. Nanotechnology: Science and Manufacturing (2015)

[8] V. Panov Influence of nanosized alloying additives on the structure and properties of powder carbon steels. Nanotechnology: Science and Manufacturing (2015) 